\title{
Mapas de zonas de risco de epidemias e zoneamento agroclimático para o Cancro Cítrico no Estado de São Paulo.
}

\author{
Mariana Vilela Lopes ${ }^{1,2}$; Modesto Barreto ${ }^{1}$, Érika Auxiliadora Giacheto Scaloppi ${ }^{3}$, José Carlos Barbosa ${ }^{4}$, Orivaldo \\ Brunini ${ }^{5}$.
}

${ }^{1}$ Departamento de Fitossanidade, Faculdade de Ciências Agrárias e Veterinárias/Universidade Estadual Paulista, V.A. Prof. Paulo D. Castellane, s/n, 14.884-900, Jaboticabal, SP, Brasil. E-mail: marianavlopes@ yahoo.com.br; ${ }^{2}$ Doutoranda em Produção Vegetal; ${ }^{3}$ APTA/ IB Rodovia Heitor Penteado, Km 3, CEP 13001-970 Campinas-SP; ${ }^{5}$ Departamento de Ciências Exatas, Faculdade de Ciências Agrárias e Veterinárias/Universidade Estadual Paulista, V.A. Prof. Paulo D. Castellane, s/n, 14.884-900, Jaboticabal, SP, Brasil. ${ }^{5}$ Instituto Agronômico de Campinas - IAC/APTA/ SAA - Caixa Postal 28, 13001-970 Campinas-SP.

Autor correspondente: Mariana Vilela Lopes.

Data de chegada: 15/06/2007. Aceito para publicação em: 23/10/2008

1501

\section{RESUMO}

Lopes, M.V.; Barreto, M.; Scaloppi, É. A. G.; Barbosa, J.C.; Brunini O. Mapas de zonas de risco de epidemias e zoneamento agroclimático para o Cancro Cítrico no Estado de São Paulo. Summa Phytopathologica, v.34, n.4, p.349-353, 2008

O cancro cítrico, causado pela bactéria Xanthomonas axonopodis pv. citri Valterin et alii 1995, é uma doença conhecida mundialmente e sempre constituiu séria ameaça para a citricultura brasileira. O objetivo do presente trabalho foi analisar as condições climáticas do Estado de São Paulo e desenvolver mapas de zonas de maior risco de epidemias de cancro cítrico. Foram utilizados dados meteorológicos referentes aos anos de 2002 a 2005, os quais foram baseados no modelo de previsão desenvolvido por Campbell \& Madden (4) e Hau \& Kranz (10). A freqüência dos dados foi horária e quando alguma estação apresentava falha, esses eram extrapolados da estação mais próxima. Foram contabilizados os índices de favorabilidade e posteriormente calculadas as porcentagens de dias favoráveis à ocorrência da doença no período de um ano. A partir destas informações, foram gerados os mapas temáticos do Estado de São Paulo, com a distribuição espacial da porcentagem de dias favoráveis à ocorrência de cancro cítrico. A região Noroeste do Estado foi a que apresentou a maior porcentagem de dias favoráveis à ocorrência de cancro cítrico.

Palavras chave: Citros, Xanthomonas axonopodis pv. citri, geofitopatologia

\section{ABSTRACT}

Lopes, M.V.; Barreto, M.; Scaloppi, É. A. G.; Barbosa, J.C.; Brunini O. Maps of zones of risk epidemics and agriculture climatical zoning of the citrus canker in the State of São Paulo.Summa Phytopathologica, v.34, n.4, p.349-353, 2008

The citrus canker, caused by the bacterium Xanthomonas axonopodis pv. citri Valterin et alii 1995, is a known disease world-wide known and it is always a serious threat for the brazilian citriculture. The objective of the present study was to analyze the climatic conditions of the State of São Paulo in order to develop zone maps of great risk for citrus canker epidemics. They had been used given meteorological referring from the years 2002 to 2005, which had been used in the model of forecast of citrus canker developed by Campbell and Madden in 1990 and Hau and Kranz in 1990. The data frequency was in hourly and when some metheorological station showed problems in the data series, these had been surpassed of the nearest station. After the accounting of the indexes had been calculated the percentages of days favorable to the occurrence of the disease in the period of one year. From this information, the thematic maps of the state of São Paulo had been generated, with the space distribution of the percentage of days favorable to the occurrence of citrus canker. The region the northwest region of the state was the one that presented the greatest percentage of days favorable to the occurrence of the disease.

Keywords: Citrus, Xanthomonas axonopodis pv. citri, Geofithopathology.

O cancro cítrico, causado pela bactéria Xanthomonas axonopodis pv. citri, é uma doença conhecida mundialmente e sempre constituiu séria ameaça para a citricultura brasileira. A doença tem sua origem no sudeste Asiático e o primeiro relato no continente americano foi em 1910 nos Estados Unidos da América, onde foi introduzida através de mudas trazidas do Japão.

No Brasil, foi constatada pela primeira vez na região de Presidente Prudente, SP, em 1957 e, embora tenham sido tomadas medidas para a erradicação da doença, a mesma se disseminou para várias regiões citrícolas e não citrícolas brasileiras atingindo os Estados do Paraná,
Mato Grosso do Sul, Mato Grosso, Rio Grande do Sul, Santa Catarina, Minas Gerais e Goiás. $(18,19)$

Segundo Massari (15), a ocorrência de cancro cítrico no Estado de São Paulo possui três marcos distintos. O primeiro, de 1957 a 1961, quando foram eliminadas 1,2 milhões de árvores. O segundo, em 1979, quando a doença foi encontrada em pomares localizados na grande região produtora do Estado, e o terceiro, em 1996, com a ocorrência da doença em viveiros da região de Limeira e com o surgimento da larva minadora dos citros (Phyllocnistis citrella Stainton, 1856) que contribuiu ao rápido aumento de focos da doença. 
A distribuição espacial do cancro cítrico mudou de padrão fortemente agregado que predominou de 1957 a 1995, para padrões de agregação intermediária, com a presença de novos focos longe do foco inicial. Esta mudança se deve provavelmente à presença da larva minadora dos citros (1).

As condições para a sobrevivência da bactéria são alta umidade e temperatura entre 20 a $39^{\circ} \mathrm{C}$. A bactéria não sobrevive por longos períodos no solo, restos de cultura ou plantas invasoras e na ausência de plantas cítricas a população diminui rapidamente no solo $(7,18)$.

A influência do clima nas doenças se faz principalmente sob a influência de períodos de molhamento favoráveis à ocorrência de epidemias e de altas temperaturas $(2,3)$. Uma rede de estações meteorológicas pode servir de base para elaborar mapas de zonas de risco de epidemias para definir um zoneamento agroclimático $(5,17)$. No caso de doenças de plantas, os mapas de zonas de risco acoplados aos modelos de simulação, podem ser úteis para indicar áreas geográficas ou até mesmo, épocas do ano mais favoráveis à ocorrência de epidemias.

Sistemas podem ser utilizados para verificar se as condições meteorológicas estão favoráveis à ocorrência de infecção pelos patógenos. Normalmente são baseados na combinação da ocorrência de fatores biológicos e meteorológicos que afetam o desenvolvimento da doença em plantas $(8,9,12,14)$. Estes sistemas podem ser usados para prever o tempo provável de início ou de aumento rápido da doença, ou então para identificar períodos em que os patógenos estão relativamente inativos $(4,20)$.

Neste sentido, o objetivo do presente trabalho foi analisar as condições climáticas do Estado de São Paulo e desenvolver mapas de zonas de maior risco de epidemias de cancro cítrico.

\section{MATERIAL E MÉTODOS}

A área estudada foi de $248.808,8 \mathrm{~km}^{2}$, onde está situado o Estado de São Paulo. Desta área foram analisados os dados de 27 estações meteorológicas pertencentes ao Instituto Agronômico de Campinas (IAC), conforme apresentado na Tabela 1.

Foram utilizados dados meteorológicos referentes aos anos de 2002 a 2005, os quais foram utilizados no modelo de previsão desenvolvido por Campbell \& Madden (4) e Hau \& Kranz (10).

A frequiência de coleta dos dados foi horária e quando alguma estação apresentava falha na série de dados, esses eram extrapolados da estação mais próxima. Os dados cartográficos para a geração dos mapas foram obtidos junto ao IBGE (Instituto Brasileiro de Geografia e Estatística). A malha utilizada para a geração dos mapas foi a do Estado de São Paulo com todos os seus municípios.

Para a geração dos valores de favorabilidade foram utilizados os modelos de Campbell \& Madden (4) e Hau \& Kranz (10), cujo modelo gera a seguinte equação: $\mathrm{Y}=\mathrm{b}_{1}\left\{\left(\mathrm{~T}-\mathrm{b}_{2}\right)^{\mathrm{b3}}\left(\mathrm{b}_{4}-\mathrm{T}\right)^{\mathrm{b} 5} \mathrm{~b}_{6}\left[1-\mathrm{b}_{7} \exp \left(-\mathrm{b}_{8} \mathrm{M}\right)\right]\right\}$, onde

Y=Severidade da doença (considerado como Índice de Favorabilidade)

$\mathrm{T}=$ Temperatura

$\mathrm{M}=$ Molhamento foliar

$\mathrm{b}_{1}=0,02 ; \mathrm{b}_{2}=11,9 ; \mathrm{b}_{3}=0,99 ; \mathrm{b}_{4}=40,0 ; \mathrm{b}_{5}=0,31 ; \mathrm{b}_{6}=1,10 ; \mathrm{b}_{7}=0,18$; $\mathrm{b}_{8}=0,07$ determinados de acordo com Dalla Pria et al. (6) para laranja da variedade Pêra.

Através do programa Visual Basic, foram contabilizados os valores diários de favorabilidade para todos os municípios, que variavam de 0 a 0,6 , sendo considerados favoráveis os índices maiores que 0,3 . Após a contabilização dos índices, foram calculadas as porcentagens de dias
Tabela 1. Localização das Estações Meteorológicas no Estado de São Paulo.

\begin{tabular}{|c|c|c|c|}
\hline Município & Latitude & Longitude & Altitude (m) \\
\hline 1. Adamantina & $21^{\circ} 41^{\prime} \mathrm{Sul}$ & $51^{\circ} 50^{\prime}$ Oeste & 443 \\
\hline 2. Atibaia & $23^{\circ} 07^{\prime}$ Sul & $46^{\circ} 34^{\prime}$ Oeste & 805 \\
\hline 3. Auriflama & $20^{\circ} 41^{\prime} \mathrm{Sul}$ & $50^{\circ} 34^{\prime}$ Oeste & 420 \\
\hline 4. Bragança Paulista & $22^{\circ} 58^{\prime} \mathrm{Sul}$ & $46^{\circ} 33^{\prime}$ Oeste & 804 \\
\hline 5. Campinas & $22^{\circ} 54^{\prime} \mathrm{Sul}$ & $47^{\circ} 05^{\prime}$ Oeste & 669 \\
\hline 6. Cananéia & $25^{\circ} 01^{\prime} \mathrm{Sul}$ & $47^{\circ} 56^{\prime}$ Oeste & 10 \\
\hline 7. Jacupiranga & $24^{\circ} 41^{\prime} \mathrm{Sul}$ & $48^{\circ} 00^{\prime}$ Oeste & 200 \\
\hline 8. Jales & $20^{\circ} 16^{\prime} \mathrm{Sul}$ & $50^{\circ} 34^{\prime}$ Oeste & 484 \\
\hline 9. Jaú & $22^{\circ} 17^{\prime}$ Sul & $48^{\circ} 34^{\prime}$ Oeste & 580 \\
\hline 10. Jundiaí & $23^{\circ} 12^{\prime} \mathrm{Sul}$ & $46^{\circ} 53^{\prime}$ Oeste & 715 \\
\hline 11. Limeira & $22^{\circ} 32^{\prime} \mathrm{Sul}$ & $47^{\circ} 27^{\prime}$ Oeste & 639 \\
\hline 12. Mandurí & $23^{\circ} 00^{\prime} \mathrm{Sul}$ & $49^{\circ} 19^{\prime}$ Oeste & 700 \\
\hline 13. Miracatú & $24^{\circ} 17^{\prime} \mathrm{Sul}$ & $47^{\circ} 28^{\prime}$ Oeste & 25 \\
\hline 14. Mococa & $21^{\circ} 28^{\prime} \mathrm{Sul}$ & $47^{\circ} 01^{\prime}$ Oeste & 665 \\
\hline 15. Monte Aprazível & $20^{\circ} 46^{\prime} \mathrm{Sul}$ & $48^{\circ} 39^{\prime}$ Oeste & 480 \\
\hline 16. Nova Odessa & $22^{\circ} 47^{\prime} \mathrm{Sul}$ & $47^{\circ} 27^{\prime}$ Oeste & 541 \\
\hline 17. Pindamonhangaba & $22^{\circ} 55^{\prime} \mathrm{Sul}$ & $45^{\circ} 27^{\prime}$ Oeste & 560 \\
\hline 18. Pindorama & $21^{\circ} 13^{\prime} \mathrm{Sul}$ & $48^{\circ} 53^{\prime}$ Oeste & 562 \\
\hline 19. Ribeirão Preto & $21^{\circ} 11^{\prime} \mathrm{Sul}$ & $47^{\circ} 48^{\prime}$ Oeste & 621 \\
\hline 20. Santa Fé & $20^{\circ} 13^{\prime} \mathrm{Sul}$ & $50^{\circ} 57^{\prime}$ Oeste & 357 \\
\hline 21. São Roque & $23^{\circ} 32^{\prime} \mathrm{Sul}$ & $47^{\circ} 08^{\prime}$ Oeste & 850 \\
\hline 22. Sumaré & $22^{\circ} 50^{\prime} \mathrm{Sul}$ & $47^{\circ} 16^{\prime}$ Oeste & 547 \\
\hline 23. Tatuí & $23^{\circ} 22^{\prime} \mathrm{Sul}$ & $47^{\circ} 52^{\prime}$ Oeste & 600 \\
\hline 24. Tietê & $23^{\circ} 07^{\prime} \mathrm{Sul}$ & $47^{\circ} 43^{\prime}$ Oeste & 538 \\
\hline 25. Ubatuba & $23^{\circ} 27^{\prime}$ Sul & $45^{\circ} 04^{\prime}$ Oeste & 008 \\
\hline 26. Vargem & $22^{\circ} 54^{\prime} \mathrm{Sul}$ & $46^{\circ} 25^{\prime}$ Oeste & 835 \\
\hline 27. Votuporanga & $20^{\circ} 25^{\prime} \mathrm{Sul}$ & $49^{\circ} 59^{\prime}$ Oeste & 505 \\
\hline
\end{tabular}

favoráveis à ocorrência da doença no período de um ano. A partir destas informações, foram gerados os mapas de zonas de risco do Estado de São Paulo, com a distribuição espacial da porcentagem de dias favoráveis à ocorrência de cancro cítrico.

Para a geração dos mapas foi utilizado o programa MapInfo ${ }^{\circledR}$ Professional versão 7.0.

\section{RESULTADOS E DISCUSSÃO}

As porcentagens de dias favoráveis, para todas as estações, durante quatro anos e a média, estão apresentados na Tabela 2.

De acordo com os dados da Figura 1, observa-se que, o ano de 2003 possuía maior porcentagem de dias favoráveis à incidência do cancro cítrico, quando comparado aos demais anos, fato este, que também foi observado através dos levantamentos amostrais realizados pelo FUNDECITRUS (Fundo de Defesa da Citricultura). No ano de 2004 houve uma menor porcentagem de dias favoráveis, próximo a $56 \%$, o que não pode ser considerado um valor baixo para a doença estudada. No ano de 2005 observa-se um aumento na porcentagem de dias favoráveis a incidência do cancro cítrico.

Na média dos quatro anos analisados, a região Noroeste mais a região de Pindorama foram as que apresentaram maiores porcentagens de dias favoráveis à ocorrência do Cancro cítrico, sendo que, tal fato já havia sido observado por Dalla Pria (6)

Nestas regiões as temperaturas são mais altas do que as demais 
Tabela 2. Porcentagem de dias favoráveis à ocorrência de Cancro cítrico (Xanthomonas axonopodis pv. citri) no Estado de São Paulo.

\begin{tabular}{llllll}
\hline CIDADES & $\mathbf{2 0 0 2}$ & $\mathbf{2 0 0 3}$ & $\mathbf{2 0 0 4}$ & $\mathbf{2 0 0 5}$ & Média \\
\hline 1. Adamantina & 78,37 & - & - & - & 78,37 \\
2. Atibaia & 41,38 & 52,87 & 39,47 & 47,67 & 45,35 \\
3. Auriflama & 65,29 & 85,08 & 78,07 & 88,49 & 79,23 \\
4. Bragança & 41,06 & 37,44 & 37,68 & 32,60 & 37,19 \\
5. Campinas & 65,25 & 61,78 & 56,77 & 58,63 & 60,61 \\
6. Cananeia & 79,76 & 82,94 & 65,96 & 57,96 & 71,65 \\
7. Jacupiranga & - & - & 60,83 & - & 60,83 \\
8. Jales & - & 70,51 & 72,73 & 78,90 & 74,05 \\
9. Jaú & 56,36 & - & 50,51 & - & 53,43 \\
10. Jundiaí & 49,33 & - & 38,39 & - & 43,86 \\
11. Limeira & - & - & 45,38 & - & 45,38 \\
12. Mandurí & - & - & 34,40 & - & 34,40 \\
13. Miracatú & 78,32 & 68,86 & - & - & 73,59 \\
14. Mococa & 74,86 & 72,81 & 57,42 & 51,43 & 64,13 \\
15. Monte Aprazível & - & 73,90 & 68,95 & 68,49 & 70,45 \\
16. Nova Odessa & 60,88 & 50,30 & 47,38 & 53,42 & 52,99 \\
17. Pindamonhangaba & - & - & - & 33,70 & 33,70 \\
18. Pindorama & - & 75,92 & 89,47 & - & 82,69 \\
19. Ribeirão Preto & 69,69 & 60,85 & 54,51 & - & 61,68 \\
20. Santa Fé & - & 92,77 & 86,62 & 92,60 & 90,66 \\
21. São Roque & - & - & 21,29 & - & 21,29 \\
22. Sumaré & - & 45,60 & 41,89 & 46,58 & 44,69 \\
23. Tatuí - & 42,98 & 41,40 & - & 42,19 & \\
24. Tietê - & 44,29 & - & - & 44,29 & \\
25. Ubatuba & - & - & 82,25 & 79,18 & 80,71 \\
26. Vargem & 43,89 & 47,43 & 33,16 & 41,92 & 41,60 \\
27. Votuporanga & 86,74 & 85,21 & 79,14 & 81,22 & 83,08 \\
\hline & & & & &
\end{tabular}

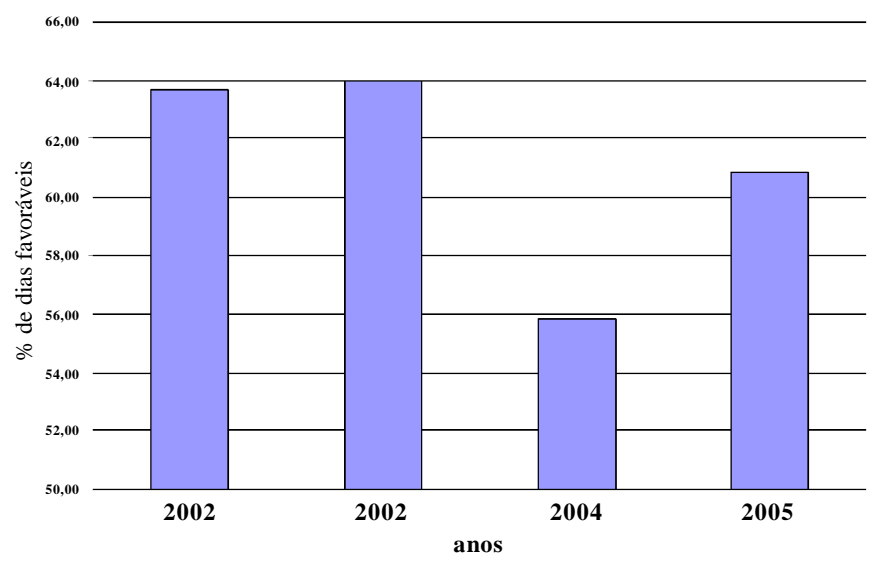

Figura 1. Médias anuais da porcentagem de dias favoráveis a incidência de cancro cítrico no Estado de São Paulo.

regiões do Estado, o que pode ser melhor visualizado nos mapas das Figuras 2, 3, 4, 5 e 6.

Na Figura 7 verifica-se um aumento significativo no ano de 2005 , quando comparado ao ano anterior, nas cidades de Auriflama, Jales e Santa Fé, localizadas na região Nordeste do Estado. Sumaré também apresentou um aumento na porcentagem de dias favoráveis a ocorrência

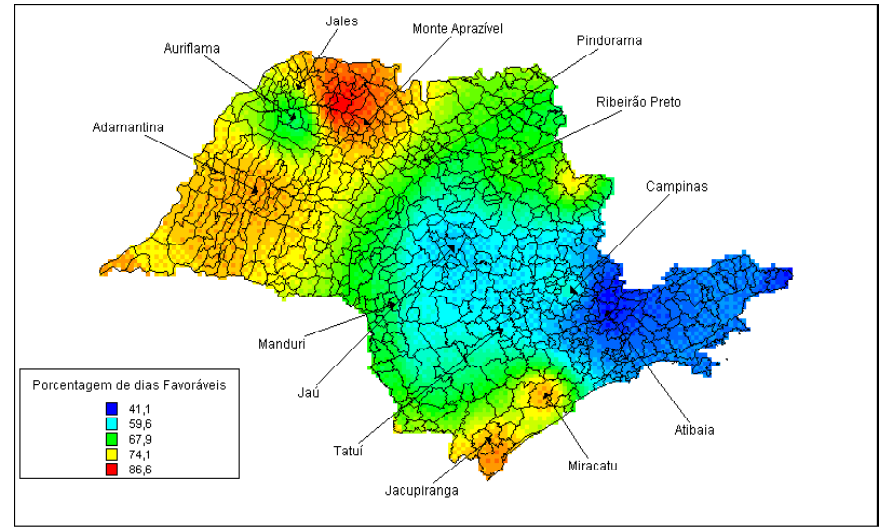

Figura 2. Porcentagem de dias Favoráveis à ocorrência de Cancro cítrico no Estado de São Paulo durante o ano de 2002.

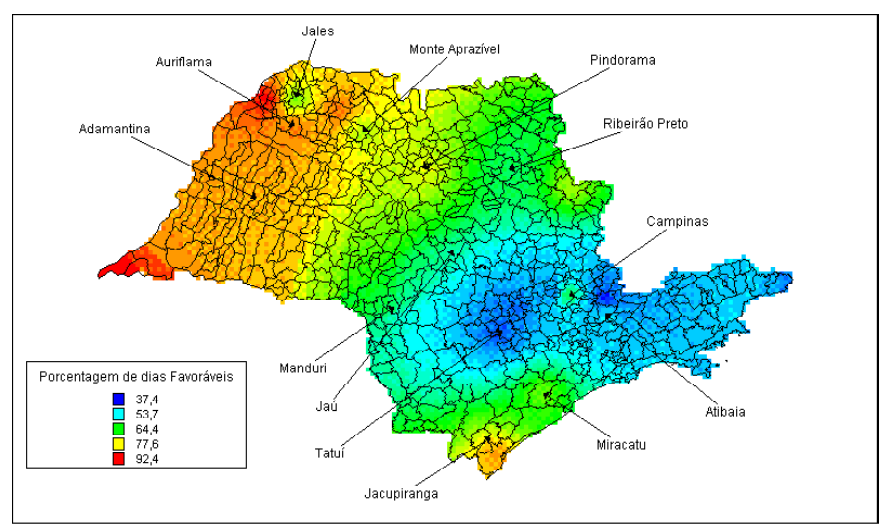

Figura 3. Porcentagem de dias Favoráveis à ocorrência de Cancro cítrico no Estado de São Paulo durante o ano de 2003.

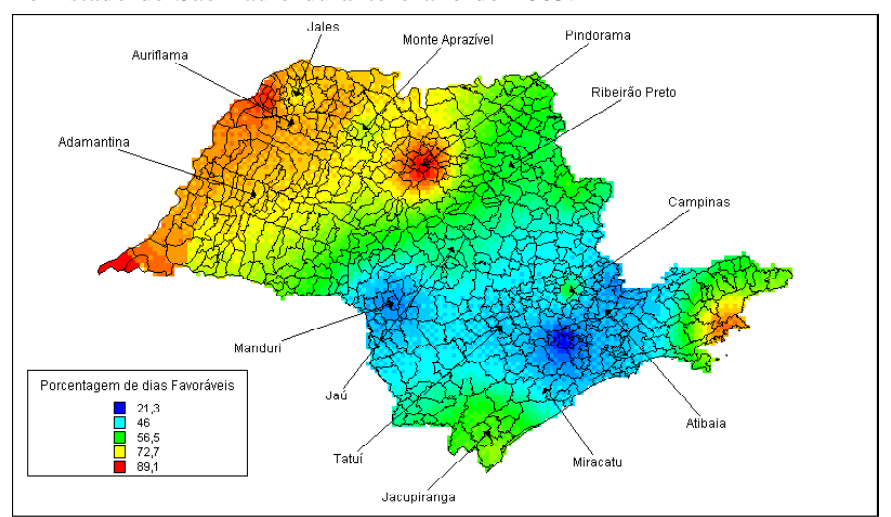

Figura 4. Porcentagem de dias Favoráveis à ocorrência de Cancro cítrico no Estado de São Paulo durante o ano de 2004.

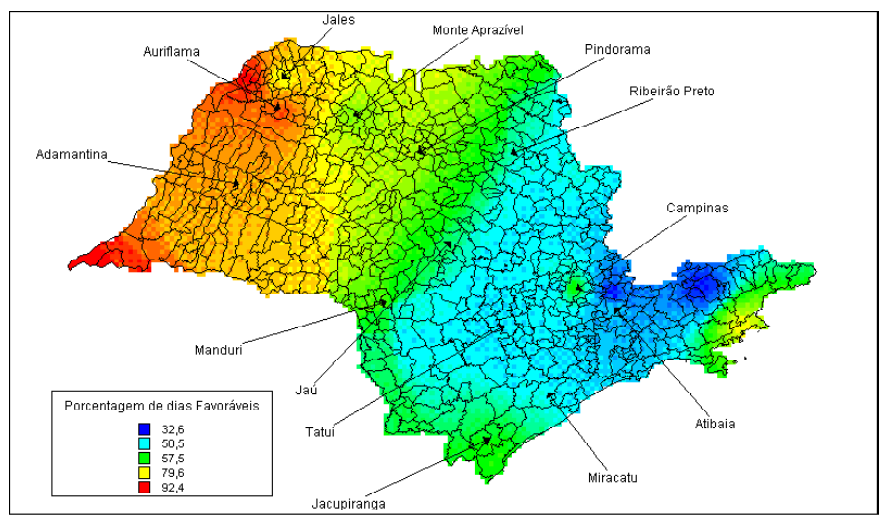

Figura 5 - Porcentagem de dias Favoráveis à ocorrência de Cancro cítrico no Estado de São Paulo durante o ano de 2005. 


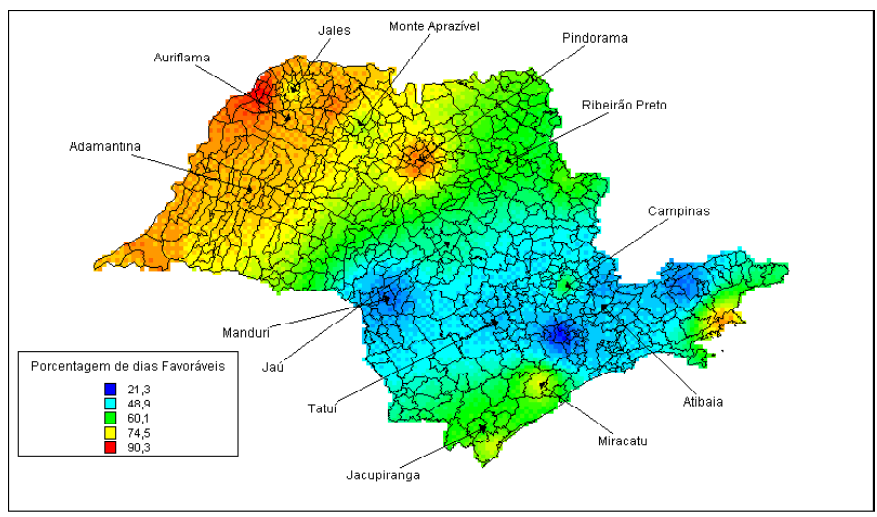

Figura 6 - Porcentagem de dias Favoráveis à ocorrência de Cancro cítrico no Estado de São Paulo. Média dos anos de 2002 a 2005.

da doença, sendo esta considerada uma região citrícola de grande potencial para o desenvolvimento da doença.

De acordo com levantamentos amostrais realizados pelo FUNDECITRUS (Fundo de Defesa da Citricultura), a região próxima ao Rio Tietê, Noroeste do Estado de São Paulo, é o local onde foram encontrados os maiores focos da doença, porém, esta região passou a ser amostrada apenas a partir de 2003.

A utilização de mapas de zonas de risco climático foi estudado por Prado (16) que elaborou uma metodologia para a confecção destes mapas para a cultura do tomateiro. García (13) também estudou o risco climático de ocorrência da Requeima da batata na região dos Andes na Venezuela.

Os dados climáticos diários, cada vez mais se tornam necessários nos estudos agrometeorológicos. No entanto, séries históricas de dados diários de precipitação, temperatura e umidade relativa não estão disponíveis ou quando estão apresentam falhas ou dados faltantes, os quais limitam o uso dessa importante informação (11).

Para uma maior precisão, é necessária análise de bases de dados mais amplas, de preferência de períodos com pelo menos 30 anos, para a criação de um zoneamento permanente de modo que seja uma ferramenta de planejamento (16).

No caso específico da citricultura estes mapas de zonas de risco podem ser muito úteis em casos de expansão de áreas de plantio, assim como nas migrações, como é a tendência atual em função da necessidade de substituição de porta-enxerto para controle da Morte Súbita dos Citros (MSC). Normalmente os novos portaenxertos necessitam de irrigação ou plantio em regiões com maior pluviosidade. Assim, seria de grande utilidade os mapas de zonas de risco, mesmo fora da região tradicionalmente citrícola.

\section{REFERÊNCIAS BIBLIOGRÁFICAS}

1. Amorim, L.; Bergamin Filho, A. A epidemiologia do cancro cítrico. Summa Phytopathologica, Jaboticabal, v.27, n.1, p.151156, 2001

2. Bergamin Filho, A.; Amorim, L. Doenças de plantas tropicais: epidemiologia e controle econômico. São Paulo, Agronômica Ceres, 1996. 299p.

3. Berger, R.D. Description and application of some general models for plant disease epidemics. In: Leonard, K.J.; Fry, W.E. Plant disease epidemiology - genetics, resistance and management. New York: Macmillan, 1989, v.2, p.125-49.

4. Campbell, C.L.; Madden, L.V. Introduction to plant disease epidemiology. New York: John Willey, 1990. p.329-52.

5. Caramori, P.H.; Oliveira, D.; Faria, R.T. Sistema de Monitoramento Agroclimático do Estado do Paraná In: Congresso Brasileiro de Meteorologia, 1994, Belo Horizonte-MG. Anais do VIII Congresso Brasileiro de Meteorologia. Belo Horizonte-MG, 1994.

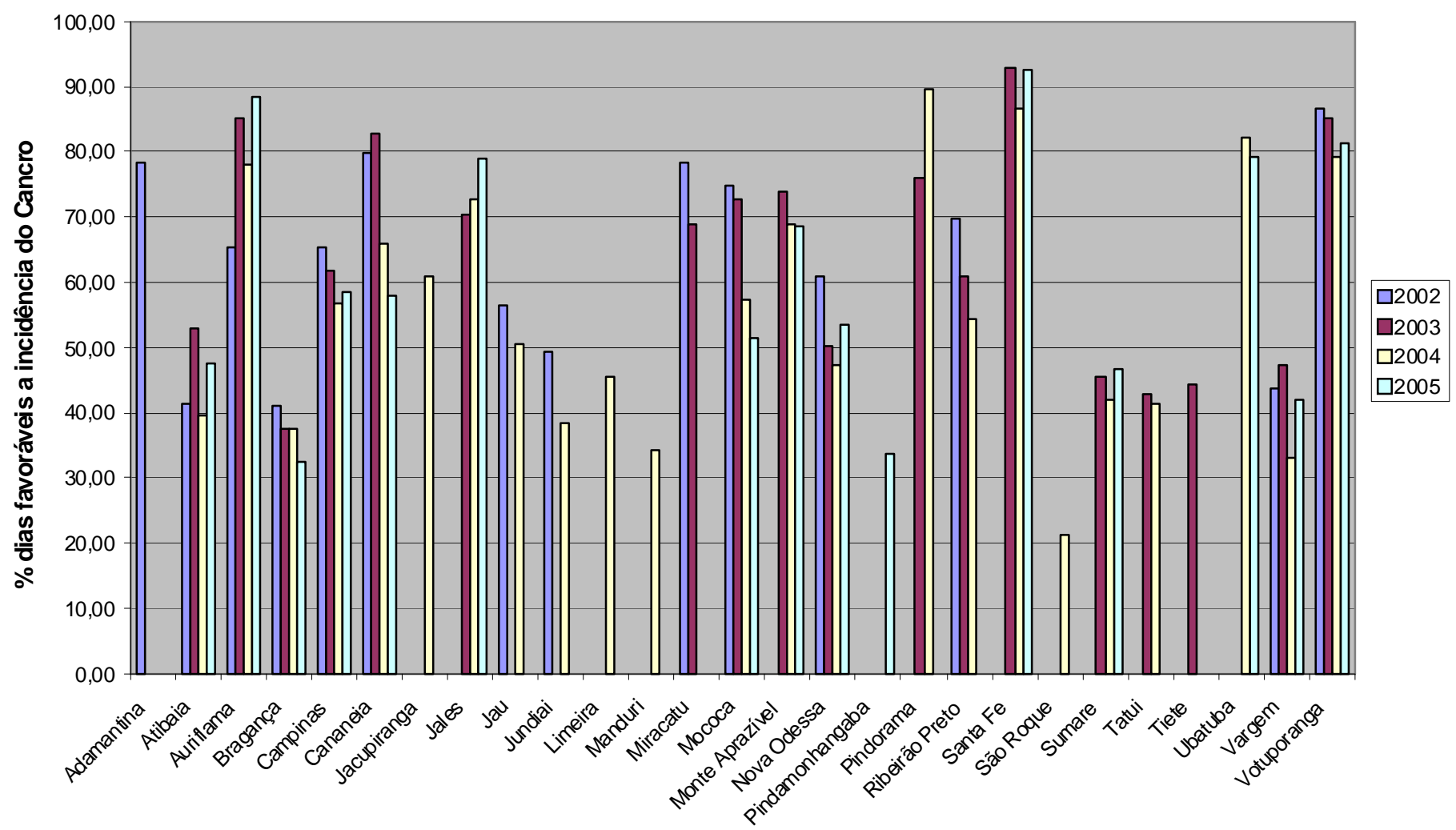

Municípios

Figura 7 - Porcentagem de dias favoráveis a incidência de cancro cítrico no Estado de São Paulo durante os anos de 2002 a 2005 por município. 
6. Dalla Pria, M.D.; Christiano, R.C.S.; Furtado, E.L.; Amorim, L.; Bergamin Filho, A. Effect of temperature and leaf wetness duration on infection of sweet oranges by Asiatic citrus canker. Plant Pathology, Oxford, Inglaterra, v. 55, p.657-663, 2006.

7. Feichtenberger, E.; Muller, G.W.; Guirado, N. Doenças dos citros In: Kimati, H.; Amorim, L.; Bergamin Filho, A.; Camargo, L.E.A.; Rezende, J.A.M. (Ed) Manual de fitopatologia: doenças de plantas cultivadas. 3. ed. São Paulo: Ceres, 1997, v.2, p.261296.

8. Fernandes, J.M. Manejo de pragas e doenças e a superestrada da informação: situação atual e perspectivas. In: Seminário Sul-brasileiro de informática na agricultura. Passo Fundo, 1996. v.1. p.24-29.

9. Fry, W.E. Disease forecasting: Epidemiological considerations. In: Fry, W.E. Principles of plant disease management. New York, Academic Press, 1982. p.105-25.

10. Hau, B.; Kranz, J. Mathematics and statistics for analysis in epidemiology. In: Kranz J., (Ed.). Epidemics of plant diseases. Mathematical analysis and modeling. Berlin: Springer, 1990. p.12-52.

11. Jeffrey, S.J.; Carter, J.O.; Moodie, K.B.; Beswick, A.R. Using spatial interpolation to construct a comprehensive archive of Australian climate data. Environmental Modeling \& Software, Oxford, v.16, p. 309-330, 2001.

12. Jonhson, K.B. The role of predictive systems in disease management. In: TENG, P.S. Crop loss assessment and pest management. American Phytopathological Society, St. y
Paul, MN, USA. 1987. 176-90 p

13. Garcia, B.I.L. Risco climático de ocorrência da requeima da batata na região dos Andes, Venezuela. 2005. 132 f., Tese (Doutorado em Física do Ambiente Agrícola) - Universidade de São Paulo, Piracicaba.

14. Krause, R.A.; Massie, L.B.; Hyre, R.A. Blitecast: a computerized forecast of potato late blight. Plant Disease Reporter, v.59, p.95-98, 1975.

15. Massari, C.A. Cancro cítrico. Fitopatologia Brasileira, Brasília, v.26, p.248, 2001.

16. Prado, T.A. Metodologia para o zoneamento de risco climático de ocorrência de requeima do tomateiro. 2006. $53 \mathrm{f}$. Trabalho de Graduação (Agronomia) - Universidade Estadual Paulista, Jaboticabal.

17. Pugsley, L., Cruvinel, P.E., Caramori, P.H. New agroclimatic digital images classification system and risk zone mapping. In: Brazilian Symposium on Computer Graphics and Image Processing, Campinas, 14; 2001. p.237-244.

18. Rossetti, V.V. Manual ilustrado de doenças dos citros. Piracicaba: Fealq/Fundecitrus, 2001. 207 p.

19. Schubert, T.S; Rizvi, S.A.; Sun, X.; Gottwald, T.R.; Graham, J.H.; Dixon, W.N. Meeting the challenge of eradicating citrus canker in Florida-again. Plant Disease, St. Paul, v. 85, n.4, p. 341 $356,2001$.

20. Travis, J.W.; Latin, R.X. Development, implementation, an adoption of expert systems in plant pathology. Annual Review of Phytopathology, Palo Alto, v.19, p.343-60, 1991. 